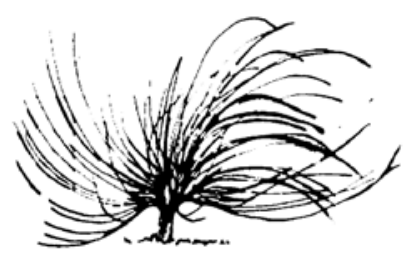

\title{
El uso del número en el proceso de enseñanza-aprendizaje con niñez de cinco años en la Institución Educativa Peruana 1094
}

\author{
Liliana Beatriz Ortiz Ortiz ${ }^{1}$ \\ Institución Educativa 1094 Jacaranda \\ Distrito de Magdalena del Mar, Lima, Perú \\ linaoortizz2010@gmail.com \\ Alejandro Cruzata-Martínez $z^{2}$ \\ Universidad San Ignacio de Loyola, Lima, Perú \\ alejandrocruzatamartinez@yahoo.es
}

\begin{abstract}
Resumen
Este artículo presenta los principales resultados de una investigación de maestría sobre estrategias vivenciales para el uso del número en el proceso de enseñanza aprendizaje de la educación inicial con niñez de 5 años. Metodológicamente corresponde al enfoque cualitativo educacional de tipo aplicada. Se aplicaron como instrumentos de investigación la entrevista a docentes y padres y madres de familia y la observación a docentes y a niños y niñas. Para el procesamiento de la información se emplearon fichas de análisis documental y la triangulación.
\end{abstract}

\section{(c) $(\mathrm{i})(\mathrm{F})$}

http://dx.doi.org/10.15359/rep.12-1.7

Recibido: 8 de julio de 2016-Aprobado: 9 de mayo de 2017

1 Profesora de la Educación Inicial en la Institución Educativa 1094 Jacaranda, en Lima, Perú. Realizó y culminó con éxito estudios de Maestría Educación, mención Investigación e Innovación Curricular en Educación Inicial en la Universidad San Ignacio Loyola.

2 Profesor-Investigador, Vicerrectorado de Postgrado,Escuela de Postgrado, Universidad San Ignacio de Loyola - USIL. ORCID: https://orcid.org/ 0000-0003-0104-0496. Google Scholar Citation: alejandrocruzatamartinez@gmail.com. DINA:000818391 
Los resultados evidencian contradicciones en el manejo del número y limitado aprovechamiento de su uso en el aula por parte de docentes, indiferencia ante el juego y las actividades en la escuela así como disposición para el trabajo matemático durante el juego cotidiano, capacidad de asombro, atención y disfrute por parte de los niños y niñas y conocimiento de los diversos usos del número en los padres. Como contribución a la solución de las dificultades identificadas se proponen estrategias vivenciales que favorecen el aprendizaje y práctica del número en las aulas a través de la comunicación y la cultura.

Palabras clave: enseñanza de las matemáticas, conceptos numéricos.

\begin{abstract}
The article reports on the main results of a master's degree research project about the experiential strategies for the use of the number from the process of teaching learning in the initial education in five year old children. Methodologically, it corresponds to educational qualitative approach applied projective type. In the investigation instruments like the interview were applied to teachers and family parents and the observation to teachers and children. For information processing, documentary analyses and triangulation of information were used. The results evidence that the teachers reflected contradictions in the handling of the number, besides a limited use of their use in the classroom and indifference for the game and the activities carried out by the children in the school; the children evidenced disposition for the mathematical work during the daily game, demonstrating capacity of astonishment, attention and enjoyment. As a contribution to the solution of the identified difficulties, experiential strategies that favor the learning and practice of the number in the classrooms through communication and culture are proposed.
\end{abstract}

Keywords: teaching mathematics, number concepts 


\section{Introducción}

$\mathrm{E}$ 1 Foro Educativo Mundial efectuado en Corea en 2015 reconoció la importancia de la educación en el desarrollo de las naciones y nos compromete, con carácter de urgencia, a desarrollar un proceso educativo renovado e integral. En esa perspectiva, el mismo foro alienta a que se imparta al menos un año de enseñanza preescolar de calidad, obligatoria y gratuita, que incida, además, en el acceso a la atención de la primera infancia, situación que limita las expectativas de muchos países, por los vacíos que deja en el abordaje educativo de las edades anteriores a los 5 años, sin tomar en cuenta las demandas de las familias y el avance de las escuelas de educación inicial que habilita a la niñez para un mejor desempeño en el nivel primario.

El presente estudio está dirigido a la educación inicial, específicamente a los grupos que atienden niños y niñas de 5 años, por el innegable impacto de la escolaridad en menores que, con ayuda de las prácticas de estimulación temprana y su participación en el nivel inicial, aprenden a socializar a través de las experiencias y juegos en los que desarrollan procesos como comparar, agrupar, que favorecen el aprendizaje comunicativo cultural que esta investigación propone a través del uso del número.

Lo expuesto en el estudio exige reconocer estos conocimientos intuitivos en los grupos infantiles, para afianzarlos en prácticas que partan de su cultura, en un marco de trabajo grupal que vincule las costumbres de la comunidad con su actitud resolutiva a través de estrategias con punto de partida en experiencias matemáticas practicadas naturalmente y que son parte de su pensamiento simbólico (Piaget, 1991).

Desde esta perspectiva, se analizan bases teóricas que sustentan el uso del número a través de estrategias vivenciales, tomando estas últimas como un soporte cultural que aproxima al estudiantado a su uso en contextos reales. Este estudio combina el enfoque onto-semiótico con el uso del número desde una visión etno-matemática, que recoge las formas de utilizar el número en su práctica personal, inmerso en los conocimientos culturales, usanzas y costumbres compartidas en un espacio-tiempo, conjugado con los avances tecnológicos que influyen en la vida del niño, la niña y sus familias, desde la sencillez de un acompañamiento cálido, atento, muchas veces inadvertido y el conocimiento intuitivo como cimiento de aprendizajes sólidos y 
complejos que acompañan al alumnado en la consolidación práctica del pensamiento resolutivo de una manera viva y contextualizada en su entorno.

En relación con este tema, el Ministerio de Educación de Perú (MINEDU, 2015) enfatiza que el personal docente olvida contextualizar las actividades que se proponen en clases, dado que parte de actividades gráficas o semi-gráficas como el llenado de largas planas para identificar un número, usando el pintado y marcado en hojas de aplicación, los cuales determinan una evidencia de un logro matemático que no está en el contexto estudiantil, puesto que son estrategias desvinculadas de la experiencia cotidiana y concreta; lejanas del juego y movimiento que necesita la población de educación inicial para desarrollar sus capacidades.

En concordancia, Gonzalez y Weintein (2013) enfatizan, con respecto a la práctica educativa en las escuelas, que los cuerpos docentes afianzados en su base teórica necesitan sistematizar experiencias volviéndolas accesibles a la escuela, estableciendo un nexo con los aprendizajes intuitivos que sus estudiantes traen del entorno, para enriquecer sus conocimientos en un marco útil, real y resolutivo, que favorezca la atención de la niñez partiendo de situaciones familiares.

El diseño curricular nacional de Perú (DCN 2009) se refiere a la experiencia que la población infantil comparte en el aula al utilizar material concreto y al comparar cantidades, al identificar y al establecer la relación número-cantidad; específicamente al utilizar cuantificadores en la vida real con experiencias directas, mediante el uso de material concreto, que facilita las representaciones gráficas como registros de cantidad, evidenciados con dibujos, palotes o puntos; lo que ayuda en el proceso de construcción de la noción de número para descubrir, en su uso, la connotación cardinal y ordinal que lo caracteriza.

En este contexto, la Unidad de Medición de la Calidad Educativa informa que los últimos resultados de la evaluación censal de estudiantes aplicada en todo el Perú en el $2^{\text {do }}$ grado de educación primaria en las escuelas estatales mostró en Lima Metropolitana, los siguientes resultados: solo el $34 \%$ de estudiantes logró el nivel satisfactorio, el 39.6\% estuvo en proceso y el $24 \%$ en inicio; lo que perfila un avance moderado en las competencias matemáticas. Datos que merecen una actitud reflexiva sobre si en las aulas de educación inicial se pone realmente en práctica trabajar a partir de las experiencias que ya tiene el niño o la niña y si, verdaderamente, se remite al sistema de conocimientos que 
trae de casa, para lograr sentar las bases del número, explorando su ordinalidad, cardinalidad e iniciación a la reversibilidad; si nuestras sesiones tienen una connotación lúdica para poner en marcha el resolver problemas sencillos del entorno, con actividades que inviten a contar, comparar colecciones, registrar puntajes en sus experiencias cotidianas y finalmente si realmente la escuela en el nivel inicial responde a situaciones que fortalecen su relación con el número y la cantidad.

Ante estos cuestionamientos, es preciso observar críticamente la relación entre teoría y práctica en el nivel inicial peruano, con el fin de evaluar su coherencia; es preciso retomar las raíces fundamentales, basadas en el juego y sus nuevas explicaciones científicas y crear estrategias que aproximen conocimientos mayores desde la experiencia, con la finalidad de facilitar el paso al primer grado de educación primaria, favoreciendo un proceso de transición feliz y productivo que facilite aprendizajes matemáticos sólidos, encaminados a un mejor desempeño en el nivel siguiente, con el fin de propiciar una mejoría en la calidad de los aprendizajes del nivel inicial peruano.

\section{Antecedentes}

En estos tiempos, los programas educativos apuestan por la experiencia del lenguaje matemático; por ejemplo, Chile aplica en sus programas educativos el lenguaje matemático para cuantificar su medio y enriquecer la comunicación numérica con la que la población infantil ya está en contacto desde el seno familiar; asimismo España ofrece, en su sistema educativo, asociar las demás áreas educativas a la cuantificación numérica del entorno, los grupos del preescolar van practicando el resolver situaciones problemáticas que implican la sustracción y la adición, además van incorporando el número mediante el uso formas de expresión gráfico matemática en donde utilizan registros de cantidades o números según su nivel representativo.

En Estados Unidos los programas educativos dirigidos al área matemática tienen una condición más arraigada en la comunicación numérica; en su enfoque educativo buscan dominar una amplia gama de habilidades numéricas que implican el uso del número desde el nivel simbólico hasta el signo, utilizan la comunicación con el fin de promover la interacción con otros para aclarar ideas matemáticas, usan las conexiones para favorecer los aprendizajes de la vida cotidiana, 
representan situaciones matemáticas con imágenes, materiales concretos, tablas, gráficos con números como elementos de construcción cultural, además evidencian el uso del número de manera contextual y organizada fundamentada en la comunicación verbal y gráfica.

En el Perú, el diseño curricular básico marca un período de transición en las aulas de educación inicial (DCN, 2009) con una versión más actualizada desde las rutas del aprendizaje vigentes desde el año 2012 y se inclina hacia la matematización del entorno.

A continuación, se mostrarán algunas investigaciones nacionales e internacionales referentes al uso del número, al manejo de estrategias de juego y experiencias vivenciales que apoyan la tendencia de nuestra investigación.

En el marco internacional, en México, el gobierno de Chiapas, realizó una investigación que estudia el desarrollo de las capacidades de lectura a través de proyectos vivenciales en una escuela multigrado (De la Rosa, 2012).

Con el fin de aportar al campo de las matemáticas en el nivel inicial, Núñez (2010), en España, presenta un diseño de talleres matemáticos dirigidos a la solución de problemas en estudiantes de $4 \mathrm{y}$ 5 años, este es un modelo de investigación educativa cuantitativo que promueve el diseño de innovaciones curriculares.

Dirigido a desarrollar la capacidad de atención de los niños y niñas en las matemáticas en conexión interdisciplinaria con otras ciencias, Ortiz (2014), en Ecuador, realizó una investigación educativa, de tipo cualitativa sobre las matemáticas, con una guía para favorecer el pensamiento matemático.

Estas tres investigaciones tienen en común situaciones que precisan cómo los grupos infantiles recogen los conocimientos de su entorno cultural y llegan a la escuela donde pueden aprovecharlos como insumos al interactuar en diversas áreas, en general y con las matemáticas, en particular.

En una comuna de Santiago de Chile, Mariana, Galvez, y Chacc (2006, citados por Ortiz, 2014) elaboraron una investigación basada en estudios que proponen elementos del juego, desde el enfoque comunicativo, en una experiencia realizada con niños y niñas de entre 7 y 8 años. Este estudio fue de tipo exploratorio, y asume que el juego es un medio de interacción y comunicación, potencia las habilidades sociales de colaboración, respeto y trabajo en grupo. 
Desde la realidad nacional, en la zona costera del Perú, específicamente en el Callao, Salas (2012) nos aportó una interesante propuesta de actividades con un enfoque sociocognitivo con el fundamento teórico de Piaget. Este estudio se dirigió al logro de capacidades matemáticas en los niños y niñas de 4 años en contenidos mencionados, demostrando un eficiente desempeño; sin embargo, en sus hallazgos, sugiere la transmisión de estos logros a las familiass en campañas de sensibilización que favorezcan los conocimientos y la real valoración del juego por la familia en el aprendizaje infantil.

Desde otra visión, Figueroa (2012), en un estudio de tipo descriptivo, comparativo aplicado, en la región Callao, para determinar la diferencia en la competencia matemática según género, tomó en cuenta tres dimensiones: geometría, cantidad, conteo y resolución de problemas. Concluye que no existen diferencias significativas en la dimensión de cantidad y conteo entre los niños y niñas de 5 años; descubrió la necesidad de diseñar programas de juegos en donde el niño y la niña puedan construir sus conocimientos a través de la interacción, ello plantea al personal docente, realizar investigaciones para conocer estrategias, diseños y metodología para el desarrollo de las competencias matemáticas, con el fin de revertir las cifras que indican un bajo rendimiento escolar en la educación primaria en esta área, creando estrategias que partan de la realidad de infantil y respondan a las necesidades de esta en el nivel inicial.

Asimismo, es preciso destacar las últimas publicaciones del Ministerio de Educación del Perú (2015) con la experiencia innovadora de la institución Educativa Inicial n. 1707 Casma, distrito de Buena Vista , año 2014, donde los profesores Flores, Chávez y Meza encontraron en sus estudiantes un limitado fortalecimiento de las competencias matemáticas, que demuestran dificultad al diferenciar cantidades, realizar agrupaciones, comparaciones y seriaciones.

Se demuestra, entonces, la urgente necesidad de examinar las estrategias de aprendizaje matemático en el nivel inicial, para que estas respondan a las necesidades contextuales del medio, de juego y recreación del niño y de la niña; además es prioritario realizar estudios que comprueben la necesidad de involucrar a los actores educativos en una participación comprometida con la educación, donde en primera instancia, cada docente sea capaz de renovar su práctica a través de bases teóricas, entremezcladas con las necesidades locales del medio en 
el que brinda educación y, en segunda instancia, se escuche la demanda de participación de los padres y madres en la educación, a través de talleres y reuniones que faciliten su conocimiento de las nuevas tendencias metodológicas del nivel inicial, desde donde se espera un apoyo concertado que enriquezca la participación interactuante: docente-familia-estudiante.

\section{Marco teórico}

En Perú, la educación inicial ofrece la oportunidad de favorecer el desarrollo integral del estudiantado, su característica más relevante es ofrecer un momento de inicio escolar único en la vida infantil, brindando oportunidades para el desarrollo humano, esto se apoya en las investigaciones dadas desde la sicología, la nutrición y neurociencias que señalan que los primeros años de vida son el momento ideal para la formación de la inteligencia, la socialización y la personalidad (Minedu,2015).

Según Chamorro, Ruiz Higueras, y Vecino (2005), en sus inicios, la enseñanza - aprendizaje del número en la educación inicial fue abordada a través del recitado y la escritura de la serie de los primeros dígitos hasta el diez; en esa época, en los libros se evidenciaban la forma como se debía escribir el número y con el correspondiente pintado de su relación cantidad con la intención de favorecer el recuerdo, basándose en que para aprender basta observar, reproducir y repetir Progresivamente se propuso en las guías de educación actividades que incluían clasificar y ordenar colecciones para introducir a través del conjunto la idea del número y su cantidad complementados con ejercicios que fueron tomados como preparatorios para la construcción del número. Esta vez se respondía al sistema piagetiano de formación de colecciones agrupaciones y clasificaciones que se dirigían a la clasificación, orden y correspondencia sobre la base de modelos constructivistas de la noción del número, pero que en la evidencia práctica, seguía teniendo presente modelos reproductores, que se evidenciaban en la cantidad de libros preescolares ,que aún hoy, promueven estos ejercicios en sus hojas.

Alsina (2012) recoge el término matemáticas informales, para referirse a las experiencias que se dan en la vida común de la niñez, dado que en las primeras edades se compila una gran riqueza de conocimiento sobre temas que interesan. Él expone que estas prácticas 
informales se dan recurrentemente, debido a esas predilecciones, que abarcan edades muy tempranas, aproximadamente a los 4 años, donde ya demuestran curiosidad innata; $y$, frente a aspectos cuantitativos, construyen en su ambiente sin límites una matemática intuitiva e informal, con formas de pensamiento imperfecto y en ocasiones alejada de la realidad, totalmente diferente a la de las personas adultas; construyen progresivamente el fundamento para el aprendizaje de los números en su vida, especialmente a los 5 años, cuando hay que darle un sentido numérico concreto y verbal a las actividades de clase, de acuerdo con las posibilidades y capacidades del niño y la niña, para el desarrollo de su carácter resolutivo.

Rencoret (1995) enfatiza que el sujeto infantil, a través de la experiencia directa, se va apropiando de las cualidades de los objetos de su entorno; verbalizando sus características como color, forma, tamaño, con las que irá estableciendo comparaciones, diferencias y similitudes, las que toman una connotación cuantitativa cuando, en colecciones de objetos, compara utilizando la correspondencia unívoca con la que se toma de referencia la cantidad.

El conteo se inicia como un juego vocal y divertido que progresivamente se va relacionando con una colección de objetos, en ese momento se convierte en la forma de comprobación de una cantidad; utilizando la comparación de cada elemento con un número, el niño o la niña, al jugar con el conteo y relacionarlo con los objetos, ejercita la posibilidad de conocer la cardinalidad y la ordinalidad en la serie numérica. Con respecto a este tema, De Castro (2007) enfatiza que el conteo oral es una etapa que consolida otras, puesto que entre juegos la niñez va aprendiendo la posición de cada número en la serie numérica. Así, entre los dos y los cuatro años, suele producirse el aprendizaje de las diez primeras palabras de la secuencia de conteo. En esta edad, forma colecciones de hasta cuatro objetos, reforzando casi espontáneamente la idea de cardinalidad.

Como complemento de la anterior perspectiva, Castro (2013) sostiene que el conteo es una habilidad que tiene como principios la correspondencia unívoca entre número y objetos de una colección, y la convencionalidad que el último número configura la cantidad de elementos, donde es indiferente el orden de la colección en la práctica del conteo. Este pensamiento, como notamos, es variable, porque en esta edad prima la impresión visual; sin embargo, frente a este pensamiento 
intuitivo se impone la experiencia concreta y social, siendo esta una oportunidad para el aprendizaje, por ello ejecutar ejercicios de correspondencia unívoca, como lo sugiere el autor, la práctica de la comparación, el uso de cuantificadores, además de los ejercicios de conteo favorecen el desarrollo de la cardinalidad, propiciando experiencias que concretizan el uso del número y la constancia de su cantidad, mientras el pensamiento irreversible del sujeto niño va asimilando experiencias que facilitan el paso de nuevos esquemas de aprendizaje.

El número es un construcción cultural que se inicia con el descubrimiento de la cantidad, posiblemente el ser humano al observar en su cuerpo los dedos de una mano y de la otra, fue rememorando estas diferencias o igualdades que se daban en sí mismo y en su alrededor, notando por ejemplo un árbol y otros más, que cohabitaban en su entorno, hasta llegar a una expresión especializada, como en las primeras manifestaciones gráficas del ser humano en la prehistoria. Así, en armonía con los cambios históricos, las diversas civilizaciones fueron perfeccionando su pensamiento matemático, pasando de ser un servicio dirigido a necesidades inmediatas, para surgir como un conocimiento que los llevó a explorar aprendizajes superiores. Este progreso evidente de las matemáticas y, por consiguiente, del uso del número posiblemente determinó el desarrollo del simbolismo que se inició con la denominación de cantidades, dando comienzo a la representación del número.

Desde un pensamiento más comunicativo, Freundenthal (1991, citado en Puig, 1995) conceptualiza el número desde los usos que asume en diferentes contextos, al ser utilizado como secuencia de recuento, cardinal, ordinal, medida, etiqueta, guarismo escrito, cálculo. Especificando que este uso se contextualiza con el momento y la expresión en que se utilizan.

Afirmando la conceptualización del número desde una mirada etnomatemática, basada en los aspectos culturales, Hilber y Higuita (2014) enfatizan que la práctica de la oralidad numérica de los pueblos es un conocimiento matemático, que se concreta en las actividades laborales que ayudan en la subsistencia. Estos conocimientos se encuentran situados socialmente en un espacio cultural preciso, y bajo una cosmovisión particular, son practicados convencionalmente como medio económico en los actos de trueque, compra o venta, son funcionales en su medio familiar y comunal por el carácter económico y productivo 
que favorece a sus familias y, por consiguiente, al movimiento comercial de su pueblo.

En suma, el número está supeditado al contexto de uso y el medio cultural en que se utiliza, que son variados. Ante esta realidad se espera que los sistemas educativos se preocupen porque el campo del número semántico personal sea tan amplio, que el alumnado pueda interpretarlo afortunadamente en variadas situaciones dentro del medio institucional formado por su cultura, asimismo que este uso le sea útil y funcional en su vida diaria, tanto en su dimensión formativa y personal, como posteriormente en su vida profesional.

La importancia del número en la escuela del nivel inicial, especialmente a los 5 años, es darle un sentido numérico concreto, verbal y utilitario en las actividades de clases, de acuerdo con las posibilidades, capacidades e interacciones que brinda su medio cultural a la población infantil, utilizando este espacio de enseñanza de forma motivante y resolutiva, que la ayude a desempeñarse exitosamente en su vida social.

Analizando el tiempo histórico, Chamorro (2011) deduce que el trato curricular del número en educación inicial demuestra una pobreza conceptual evidente, puesto que no responde a las reales posibilidades del estudiantado. Se exploran muy poco los fenómenos que se dan en torno al uso del número. Además se trata limitadamente el acceso al conteo y en el cálculo, tanto escrito como pensado, si bien es cierto la experiencia concreta en educación inicial es importante, el conteo es un ejercicio que facilita la secuencia numérica, los juegos comparativos basados en la correspondencia unívoca que son las bases de las construcciones matemáticas que favorecen el pensamiento resolutivo.

En respuesta a estas temáticas, los sistemas educativos nacionales inciden en programas vanguardistas basados en la experiencia concreta y resolutiva, que sugieren enfoques más actuales basados, por ejemplo, en la matemática realista, el enfoque ontosemiótico, la función del número desde habilidades de resolución de problemas; sin embargo, en el aula se vive un velo de invisibilidad al uso del número que el niño y la niña practican espontáneamente tanto en la escuela, como en el hogar. En suma, actualmente, se tienen dos tendencias educativas al desarrollar matemáticas en las aulas, una basada en la experiencia comunicacional - resolutiva desde lo concreto y otra desde las jerarquías planteadas a partir de una construcción adulta; con pasos previos para 
llegar a la noción del número; situación que merece una larga reflexión y evaluación que parta de valorar realmente los conocimientos que traen la niñez a la escuela estableciendo cuán significativa es la enseñanza que promovemos hasta hoy.

Dentro del marco de los enfoques y teorías que sustentan el aprendizaje basado en la experiencia se comparten algunas definiciones y aportes actuales sobre las estrategias vivenciales que coadyuvan hoy a la labor educativa.

Farci (2007) configura las estrategias vivenciales como trabajos de campo en donde el estudiantado tiene la oportunidad de interactuar con el ambiente y sus compañeros y compañeras, facilitando experiencias directas y sensoriales que permitan activar procesos del pensamiento investigador tales como: observación, identificación, descripción, comparación, interpretación, organización y aplicación, con relevancia en el hacer y el sentir una conexión entre lo cognitivo y lo emotivo que, unido al descubrimiento y a la cooperación, hacen que el aprendizaje sea significativo.

Desde otra tendencia que parte de un contexto matemático, Hernández (2013) enfatiza que "las experiencias que viven al interactuar con su entorno, desarrollan nociones numéricas, espaciales y temporales que les permiten avanzar en la construcción de nociones matemáticas más complejas" (p. 50).

En búsqueda del origen de experiencias que nutren las estrategias vivenciales, es pertinente nombrar como precedente los sistemas que rodean a la niñez antes de ir a la escuela, entre ellos la familia, la cultura, el espacio y el tiempo que contribuyen a formar el bagaje de recuerdos y conocimientos con el que está en contacto; como se ha explicado anteriormente, son un soporte a la educación institucionalizada porque favorecen procesos cognitivos básicos que conectan el aprendizaje con el entorno inmediato y, además, con la emoción de conocer algo nuevo con sus pares, construyendo en esa relación nuevos conceptos y habilidades que le ayudaran a enfrentarse cotidianamente a la vida.

Las estrategias vivenciales desde esta mirada recogen las experiencias socio-culturales en las que se desarrolla el ser humano desde que nace y recibe la influencia familiar y comunal; en donde desarrolla el juego como práctica simbólico cultural con el que se apropia de los comportamientos, usanzas y tradiciones del medio donde las rutinas son actividades convencionales que se afianzan en la práctica diaria de 
actividades propias de su cultura escolar, como el saludo, la formación de filas por orden de tamaño, el conteo de niñas y niños del aula, la celebración de cumpleaños, entre otros.

En nuestra experiencia, se han recopilado diferentes actividades con potencial educativo que responden a situaciones significativas en la vida del niño y la niña, estas se desarrollan espontáneamente con el juego recreando así el aprendizaje propio de su cultura. La etnomatemática es un enfoque pedagógico que valora la cultura como fuente de sabiduría popular de donde parten conocimientos que van a desencadenar otros más complejos que coadyuvan al avance práctico-cognoscitivo de una población.

El proceso de enseñanza - aprendizaje desde una mirada etnomatemática insiste en revalorar los conocimientos matemáticos del medio basado en las usanzas y costumbres para, partiendo de ellos, consolidar un aprendizaje significativo que al ser conocido por el niño o la niña, recoge elementos básicos de su cultura mediante las actividades comunicativas que responden al grupo étnico-cultural del que participa y del que extrae conocimientos personales y convencionales de origen ontosemiótico, que tienen como características fundamentales las costumbres, sus sistemas de creencias propios de su medio.

Desde esta mirada, la educación con base etnomatemática y ontosemiótica se consolida en dos miradas al número: desde su connotación comunicativa y la cultural Estas son utilizadas de manera diaria y funcional dentro de los sistemas que rodean a la niñez y de ellas recoge las formas de aplicarlo de manera resolutiva: del medio toma los recursos verbales comparativos que responden a las costumbres de su comunidad.

En este análisis del aprendizaje etnomatemático no puede faltar una mirada crítica a la actitud docente frente al espacio cultural en el que se actúa, por ello debe compenetrarse con el saber del medio en el cual enseña e identificarse con él para así proponer, a través de estos conocimientos, estrategias pedagógicas que sean efectivas en el proceso de enseñanza-aprendizaje.

\section{Metodología}

El trabajo de campo en la investigación se desarrolló en la institución educativa 1094 Jacaranda, situada en el distrito de Magdalena del Mar, una zona urbana de la Cuadra 4 del jirón Trujillo. Este trabajo permitió el diagnóstico del estado del uso del número en el proceso de 
enseñanza - aprendizaje con estudiantes de cinco años para lo cual se elaboró una matriz formada por la categoría apriorística uso del número, visto como una práctica que se origina en el actuar diario y las subcategorías:

- comunicación numérica, corresponde al uso de número respecto al uso de palabras numéricas, implica comunicación numérica en el entorno y el uso de cuantificadores.

- función del número, basada en la visión del número como cardinal, ordinal, conteo, medida, registros de cantidad y resolución de problemas.

Los instrumentos consistieron en un diario de clases que tuvo por finalidad observar a estudiantes y docentes en la escuela, así como también entrevistas una dirigida a los padres y madres y otra a docentes con el fin de diagnosticar sus opiniones y experiencias sobre el uso del número.

De forma complementaria se aplicó una guía de observación que recogió las impresiones de los docentes, niñas y niños en el ejercicio pedagógico y en las actividades libres, en cuanto al profesorado, con la finalidad de observar como utilizaban los números en las clases, y en los grupos infantiles, como los asumían en la clase y en sus momentos libres, en sus juegos. Para ambos grupos se examinó si eran aprovechadas las oportunidades para poder hacer un uso del número de forma real y contextualizada en la vida diaria del aula y otros ambientes de la comunidad, con la premisa de que el número está presente de forma transversal en la vida.

La observación se realizó en las actividades de aprendizaje de 5 años del turno tarde dentro y fuera del aula, siendo esta información registrada en 5 diarios de clase en los que se anotaron las actividades que se realizaban.

Para el procesamiento de toda la información recogida se emplearon dos técnicas cualitativas de investigación, el análisis documental y la triangulación. El análisis documental asumido como una serie de operaciones destinadas a la captación, evaluación, selección y síntesis de los mensajes subyacentes en el contenido de los documentos, a partir del análisis de sus significados, a la luz de un problema determinado, Así, contribuye a la toma de decisiones, al cambio en el curso de las acciones y de las estrategias (Bisquerra, 1996). 
La triangulación es concebida como un modo de proteger las tendencias de quien investiga, al confrontar y someter a control recíproco las respuestas de estudiantes, familia y docentes informantes participantes de la investigación (Taylor y Bogdan, 1990). Esta técnica ayudó a comparar las diversas apreciaciones, estudiar dónde difieren, están de acuerdo o en desacuerdo. Según Woods (1993), este método tiene una fuerza enorme, siempre y cuando se utilicen tres procedimientos distintos para obtenerla, en la presente investigación se emplearon: el diario de clases, las guías de observación y las entrevistas; debido a que con ello se aumenta la probabilidad de exactitud en los resultados.

En la aplicación de estos instrumentos se descubrieron, en el trabajo de campo, las categorías emergentes: la capacidad de asombro y disfrute, el trabajo en equipos, la autonomía y seguridad, comunicación de la experiencia y comunicación numérica.

Con respecto a los niños y niñas se observó la capacidad de asombro y disfrute, denotados en sus impresiones al descubrir utilidades y retos de cantidad y su posibilidad de resolverlos en las diversas dinámicas de trabajo, por ejemplo, al guardar los juguetes y ante la repartición de materiales, cantidad; la mayoría reclamaba repartir los materiales y tratar de hacer estas actividades en su grupo así como el disfrute, característica repetitiva, observada en este estudio. Esto permitió verles en función de su desempeño en actividades que contenían juegos o retos con puntajes o relaciones de cantidad con representaciones o números, y cómo se sienten a gusto. Ello se hace evidente cuando, por ejemplo, se divierten al contar las cantidades de niños que están con ellos, comentan si hay más niños o niñas y lo hacen espontáneamente en el aula o en sus juegos.

Es importante mencionar el trabajo en equipos, configurado por la necesidad de los niños y niñas por trabajar, jugar y compartir en grupos. En edades de 5 años prefieren las actividades colectivas, aunque en el aula se promueva el trabajo personal, lo que se denota en las actividades de juego donde tienden a formar grupos en el recreo.

En conexión con la anteriores categorías se denotó la autonomía y seguridad, actitud que los niños y las niñas exigen hacer en variadas actividades de aula como el repartir materiales; además usan, por iniciativa propia, su capacidad resolutiva que nace de su recursos personales aprendidos en el medio, combinando la imitación y la intuición, situación 
que muchas veces era realizada por la auxiliar de aula, limitando las posibilidades de participación de los niños y las niñas.

Asimismo, las experiencias comunicativas fueron descubiertas como categorías en lo que se llamó comunicación de la experiencia o asamblea, momento en que el niño o la niña expresa las ideas con las que se enfrenta ante diversos momentos problemáticos en su vida y las cuentan con la mayor naturalidad favoreciendo la socialización de los aprendizajes y la transferencia, estos también se notan en la comunicación numérica, donde en sus diálogos y juegos simbólicos cotidianos hacen uso de palabras con contenido de cantidad o con connotación numérica que son usadas espontáneamente para describir o comparar colecciones de objetos o situaciones. Se observó el uso de términos locales como "empate", "hartos" juguetes, "un montón", "todos arrejuntaditos", entre otros; palabras que deben ser valoradas y no excluidas del lenguaje infantil, con el fin de partir del lenguaje común del entorno social, para progresivamente acercarlo al lenguaje convencional sin negar sus usanzas y costumbres culturales.

\section{Categorías emergentes en los docentes: Rutina, contradicción entre la} teoría y la práctica, interés limitado por la innovación y el medio cultural

En cuanto al personal docente cuyo actuar pedagógico fue recogido desde los diarios y las entrevistas en las mismas categorías descritas anteriormente se descubrieron las categorías emergentes: entre ellas la rutina característica que responde a una pobre observación al actuar del niño o la niña en el aula, lo que impide ver posibilidades de trabajo creativo.

Conjugando las respuestas en las entrevistas y contrastándolas con la práctica se descubrió una contradicción entre la teoría y la práctica, situación que se muestra en la actitud docente, lo que separa le separa de una visión objetiva de su práctica pedagógica con las teorías que la sustenta, en consecuencia, se nota un discurso repetitivo que responde a situaciones determinadas y no las contextualiza o aplica en necesidades del proceso. Asimismo, se evidenció un interés limitado por innovación y el medio cultural que implica desde este un real alejamiento a reflexionar sobre estrategias nuevas u otras posibilidades de intervención fuera del aula; además, limitado interés por compenetrarse con las actividades de la comunidad que pueden impactar en el ámbito educativo, en ese 
sentido no utilizan ambientes de la comunidad en las sesiones; es interesante recalcar que este interés limitado y desconocimiento del medio cultural no era general, puesto que una profesora vive en la comunidad, sin embargo, su tendencia de aprovechar el medio cultural en la labor educativa es limitadamente utilizada en la escuela, puesto que las actividades se reducen generalmente al acuerdo de la mayoría.

\section{Categorías emergentes en los padres y madres: Atención al desarrollo matemático, expectativa ante el trabajo en la escuela, la necesidad de participación en la educación de sus niños y niñas}

Entre los padres y madres se encontró una especial atención al desarrollo matemático, dado que en forma reiterada comentaban con entusiasmo los logros de los niños y niñas en conteo de objetos, personas, manifestaciones gráficas con evidencia matemática, conteo verbal como un juego libre, comparación de medidas con instrumentos de medidas arbitrarias, como el centímetro y la romana, actividades que los niños practican en casa.

Demostraron, en las entrevistas, una marcada expectativa ante al trabajo en la escuela, y se interesan por apoyar a sus niños y niñas, manifiestan interés por comprar libros para avanzar en la escritura de los números, en aplicaciones con sumas sencillas, practicar el conteo hasta el 100 y piden a los niños y niñas hacer actividades en casa relacionadas con la cantidad y el uso del número, por ejemplo, cómo ordenar la mesa en sus hogares con la distribución de servilletas y cucharas tomando en cuenta los comensales, en el conteo de sus juguetes con objetos de apoyo en las calles, el conteo libre, interés por los números en los carteles y etiquetas que contienen números, además de logros y actividades resolutivas diversas observados en la vida cotidiana.

Ante la demanda de participación de la familia en la educación de sus hijos e hijas, surge como una categoría emergente la necesidad de participar activamente en la educación escolarizada, lo que establece un requerimiento que implica voluntad de apoyo en las actividades referentes al uso de número y una necesidad de información sobre cómo acompañar a los niños y niñas en este aprendizaje. Esto exige una responsabilidad docente de ayudar al padre o la madre en el proceso de aprendizaje con aulas abiertas y con su participación, donde se ejecuten actividades de forma demostrativa y vivencial sobre cómo aprende la niñez con actividades 
cercanas al entorno familiar, que puedan ser practicadas en la casa para que aprovechen los elementos culturales que los rodean.

\section{Análisis interpretativo y discusión de los resultados}

En cuanto al manejo del uso del número en clases se denotan muchas oportunidades para hacerlo; pero una limitada disposición para ello en situaciones como la asistencia, el momento de hacer filas, al organizar la secuencia del día, acciones que quedan como una posibilidad trunca en el aula, asimismo concluimos que se explora muy limitadamente la capacidad de comparar en el aula.

Una situación similar se observó en las actividades que dan la oportunidad de repartir, registrar y contar, las que se podrían efectuar cotidianamente; sin embargo, se ven limitadas porque las repeticiones de materiales están a cargo de la auxiliar, el registro de conteo solo es mencionado por la docente, por lo tanto, no se crean situaciones vivenciales para usar el número y su cantidad, en las actividades de aprendizaje y las actividades de rutina.

La observación, un tipo de evaluación de proceso, que en educación inicial contribuye a la evaluación final, se consideró ausente en el cuaderno anecdotario y el diario de clase no se registraba al concluir esta. De la misma manera, la disposición de observación en los momentos de juego y trabajo, así como también la socialización de los aprendizajes al término de las sesiones, y de esta dinámica de trabajo, se presentó incompleta en los días de observación a pesar que los niños y niñas tenían producciones con los "playgos" y juegos maderas, juegos con las balanzas, que podían compartir entre sí. A la primera hora de trabajo se carecía de una organización y del seguimiento de la en el proceso.

Asimismo, el personal docent presentan contradicciones en relación con el manejo de la teoría y la práctica, sus respuestas en la entrevista denotaban un conocimiento teórico que en sus aulas no se reflejaba, en consecuencia, presenta limitaciones en el tratamiento del número de forma cotidiana en las actividades diarias y permanentes, las actividades de registros y conteo no eran tomadas en cuenta como un actuar pedagógico de uso diario.

Los niños y niñas presentan grandes potenciales en el uso del número, lo utilizan naturalmente en la comparación de cantidades, el conteo, la medición, el registro el uso de ordinales es parte de un 
conocimiento intuitivo que está en constante práctica, en la escuela va más allá de las actividades programadas, por tanto, la observación y la atención de la niñez debe ser un actuar cotidiano indispensable que implique no solo registrar las sesiones de aprendizaje sino ir más allá en las actividades de clases, el recreo debe ser considerado como una posibilidad de observación que debe nutrir las estrategias de aprendizaje con las actividades que lo niños y las niñas prefieren hacer, o que se convierte en una demanda silente sobre cómo aprenden desde sus predilecciones y necesidades a lo que el personal docente decide, si contestar o no contestar desde actividades que respondan al nivel de conocimiento y necesidades de la población infantil. En términos concretos la clave es observar.

La discusión sobre las categorías emergentes se organizó, desde la triangulación de la información en función de tres temáticas fundamentales: las características y potencialidades de los grupos infantiles, las potencialidades y debilidades docentes y la demanda de los padres y madres en el grado inicial 5 años; los tres temas dentro del marco del uso del número y su connotación matemática se interpretaron desde una mirada teórico-práctica, analizando sus efectos en el uso del número en el nivel inicial.

\section{Características y potencialidades de la niñez}

Esta población infantil en el nivel inicial, como hemos podido ver en los diarios y las entrevistas, es curiosa, presta a experimentar con toda clase de instrumentos que estén cerca de su mano, gusta jugar y trabajar sola y disfruta los momentos de trabajo colectivo, se ayuda entre sí; sin embargo, también se agrede, si no hay una mirada atenta que los haga recordar sus acuerdos de convivencia; leen imágenes, preguntan y pugnan por tener la atención del sujeto adulto.

Una potencialidad en los grupos infantiles es su capacidad de resolver problemas de forma creativa, les gusta jugar, que les cuenten cuentos y saben cuándo no hay supervisión y pueden cruzar las línea de las reglas, cómo ir a jugar al baño, para mojar a los compañeros con el agua o subir las escaleras para explorar el segundo piso.

Les gusta registrar, graficar, formar colecciones y comparar quién tiene más, quién tiene menos; les gusta competir y ganar; según sus preferencias cantan, bailan, imitan a su profesora y siempre experimentan 
con palabras nuevas que van incrementando en su trato diario con las personas que les rodean, tienen gustos definidos que dicen colectivamente. Según (DIAR2) la profesora pregunta: ¿chicos que es lo que más les gustó en clase hoy? ¡comer! dijo uno y otro le dijo ¡pintar!, ¡dibujar!, mientras la profesora se sentía intimidada porque ella esperaba otra respuesta.

Sin embargo, prestan atención a todo, la sensibilidad por los sucesos que les rodean son una oportunidad para aprender, es por ello que en nuestras categorías dirigida a estas edades infantiles se ha considerado, en forma global, la emoción, entre ellas el asombro y la alegría muy presentes en el nivel inicial 5 años, producto de nuevas situaciones, que comparten con su grupo e incrementan su capacidad de disfrutarlas en forma colectiva.

\section{Capacidad resolutiva}

Los niños y las niñas naturalmente, por iniciativa personal y grupal, resuelven los conflictos en el juego y en su actuar cotidiano en el aula. Este espíritu resolutivo es incrementado cuando se les brinda la oportunidad de tomar decisiones, situación que era obviada en el aula y que frecuentemente era asumida por las personas adultas, lo que se registra en los diarios en donde según (DIAI 1) la profesora no les da la oportunidad de demostrar cómo pueden solucionar una actividad por sí mismos o mismas, repartiendo las loncheras, materiales; situaciones diarias que contribuyen a la organización del aula y a su vez a la comparación, conteo y correspondencia: así, limitan sus capacidades.

A ese respecto, es evidente que su capacidad de resolver problemas, esto es evidente en los mismos niños y niñas en los diarios docentes donde según (DIAA8) la profesora les decía, ...guardar, guardar y ella iba ayudando a ordenar en la mesa de construcción... y así también a trabajar en forma colaborativa para la solución de problemas, lo que resulta obvio según el diario: (DIAA9) donde los niños empezaron una dinámica de ayuda mutua ayudándose unos a otros por iniciativa propia, para la solución de un problema. 


\section{El trabajo colectivo en grupos}

La niñez gusta de trabajar en grupo, contestar a coro, aun cuando no se haya indicado, buscan grupos para jugar, para dramatizar, compartir sus producciones, resolver situaciones, tienen sus amigos preferidos, algunos prefieren un solo lugar dónde sentarse para compartir con su único amigo y otras prefieren cambiar de sitio donde hay más emoción y posibilidades para jugar, pero finalmente siempre requieren de sus amigos y amigas para jugar y divertirse según sus predilecciones; es evidente que en este trato hay conflictos que se suscitan, pero estos son parte de la vida y la profesora demostró que con un trato cariñoso era posible solucionar las diferencias.

\section{El juego en la niñez}

En el recojo de información del diagnóstico, el juego ha estado transversalmente presente. En las actividades docentes se observaron juegos con la pelota, con el cuerpo, con materiales didácticos, con colecciones que traían de casa; una vez más se comprueba que el juego está presente en le educación inicial; sin embargo, este resulta casi inexplorado por las docentes de las aulas; en el recreo los niños y las niñas juegan y quedan a cargo de la auxiliar, se organizan con sogas, con pelotas y en esa organización se mueven capacidades que no las vemos en clases como cantidad de pelotas, cantidad de niños que mojaron a una niña en el baño, grupos de juegos en las escaleras contando, el juego manifiesta una riqueza enorme si es observado con atención, evidente, si no somos perceptivos ante esto, no tenemos la oportunidad de determinar la inmensa cantidad de situaciones pedagógicas que convergen, por ello se propone una dinámica de juegos con talleres organizados en el recreo, evaluados como logros de proceso, con guías de observación precisa y diarios docentes como materiales complementarios, actividades que sean retroalimentadas en asamblea con los grupos infantiles. Si se sistematiza la evaluación del nivel inicial se puede alcanzar el fin de darle mayor credibilidad a los resultados para ver la calidad de impacto en el aprendizaje de la niñez. 


\section{La comunicación numérica y la niñez}

Los niños y las niñas brindan constantemente información numérica que comparten con el grupo de estudiantes, información matemática que abarca números ordinales, pesos y tamaños porque forman parte del habla local no convencional y están en contacto directo con sus usos valorativos a través de la comunicación y las vivencias. Algunos saben el valor del sol y cuidan con mucho esmero sus monedas, otros juegan a las entrevistas con diferentes instrumentos de medida. Otra actividad que les gusta mucho, es medir a sus compañeros, medir objetos, filas, medir cantidades y colecciones por su extensión, lo que los lleva experimentar con palabras numéricas o que implican cantidades, comparando colecciones, usando los cuantificadores formales o no formales; otras se interesan por la hora para ver los programas de televisión o ir a la escuela o en el momentos de la lonchera aprenden la disposición del reloj en una hora determinada, para tomar sus alimentos, así van aprendiendo a ver la hora, descubren el número en la calle y preguntan, cuentan al subir las escaleras y la serie de los semáforos en decreciente, disfrutan los números contando los carros de la avenida.

La comunicación numérica es una usanza común y muy productiva de la que la niñez se apropia en forma constante y cada vez más perfeccionada. Gay y Cole (1997, citados en Carraher y Carraher, 2004) coinciden en que es necesario conocer las matemáticas inherentes a las actividades de la vida diaria que están dentro del ámbito cultural de la niñez, con el fin de construir, a partir de ellas, puentes y ligamentos efectivos para las matemáticas más abstractas que la escuela se proyecta a enseñar.

En forma conclusiva la comunicación matemática, vista en estas dos características: la del lenguaje numérico y la información matemática, deben partir del entorno del niño y la niña, para así relacionarlas con conocimientos mayores.

\section{Las potencialidades y debilidades docentes}

\section{Actuar rutinario docente}

El actuar rutinario docente hace ver al grupo igual cada día, cada año; sin observar detalles que podrían ser parte del emprendimiento 
de nuevos proyectos o la aplicación de innovadoras estrategias que favorezcan el aprendizaje estudiantil y a la vez activen la capacidad creativa docente por adaptar o crear nuevas situaciones de aprendizaje que puedan ser retroalimentadas y renovadas en su práctica, según el grupo con el que trabaja, teniendo en cuenta que cada grupo infantil nunca es igual a otro y que la flexibilidad es importante porque permite adaptarse a diferentes situaciones o grupos.

En nuestro contexto actual, las especializaciones y cursos tienden a renovar nuestros conocimientos educativos, pero las capacitaciones no son la única alternativa contra la práctica educativa rutinaria; según lo recogido en esta realidad diagnosticada, se evidenciaron rasgos que determinan esta práctica educativa como rutinaria, en función a ello, el personal docente presenta, según el análisis global de los diarios (DIA1234) lo siguiente: se denota una limitada atención por parte del docente a la observación en los niños y niñas, así como un limitado interés en sus logros especialmente en la hora de juego y el recreo. En esta aula se mueven con mayor interés en la próxima promoción y eventos escolares, a desarrollarse, como el desfile.

Es necesario tomar en cuenta que entre el personal docente observado, uno es nombrado con estabilidad laboral y el otro no lo es, y tiene la condición de contratado, por lo cual frecuentemente es evaluado por el sistema nacional liderado por el Ministerio de Educación (MINEDU), situación que determina una capacidad probada en conocimientos; sin embargo, estos no se transfieren a su práctica educativa. Asimismo, el docente nombrado consta con capacitaciones recientes, lo que no se ve reflejado en la práctica pedagógica profesional .En cuanto a su trabajo en el turno mañana hay que tomar en cuenta que son 29 niños en aula y que la exigencia en cuanto a ruido y manejo de espacio es mucho más difícil porque se comparte patio y baños con primaria, que ostenta una mayor carga de matrícula que inicial, debido a la creciente cantidad de niños y una falta de previsión en las matriculas tanto de inicial como en primaria. Por ello ponemos en consideración analizar si es pertinente la convivencia del nivel inicial y primario en una sola escuela, aun cuando los espacios de los ambientes no favorecen el libre tránsito y los grupos infantiles juegan, corren y pueden ocasionar peligros para la integridad física por el limitado cuidado. 


\section{La cultura como motor educativo}

Desde este estudio se considera la cultura como categoría macro educativa, porque recoge las costumbres, el juegos y las actividades resolutivas que emergen como categorías y son asimiladas como parte de la cultura, donde se ha desarrollado la niñez; esta macro categoría parte de los conocimientos propios del niño y la niña y se siente en el desarrollo de las actividades desde las formas de expresión hablada, los juegos del entorno y las costumbres que abarcan las formas de cómo, desde sus saberes, resuelven problemas respondiendo a los sistemas en los que recibieron las primeras situaciones de aprendizaje por familiares (Bronfenbrenner, 2005, citado por Swick y Willians, 2006; Somoza 2003, citado por Pinto, Giron, y Villegas, 2014). Asimismo influye el lenguaje que, como una forma comunicativa, es parte de las personas y que pasa al ser convencional dentro de un espacio de tiempo y una población, por eso se asume que la escuela debe estar empapada de esa cultura, con el fin de establecer aprendizajes significativos que formen parte de los intereses de la niñez (D`Ambrosio, 2014).

\section{Las actitudes coadyuvantes}

Son aquellas que coadyuvan en el proceso de enseñanza aprendizaje, entre las que encontramos: la emoción, el asombro, el disfrute, la seguridad y autonomía, actitudes que fortalecen el aprendizaje en sí, dando nuevas oportunidades para explorar al manejo del número, en donde los niños y las niñas demuestran capacidad de asombro al descubrir una situación nueva, así como también muchas posibilidades resolutivas; además disfrutan al experimentar con situaciones nuevas o de su interés y, al hacerlo, demuestran seguridad y autonomía al enfrentarse a situaciones problemáticas de la vida diaria. Este grupo de actitudes tiene como características que se cohesionan con los aprendizajes y toman una connotación metacognitiva, porque son el soporte que motiva sus ejercicios. En este proceso es indispensable el seguimiento del personal docente con una actitud de arropo afectivo (Ugarttexea, 2001), que acompañe al niño o a la niña evitando anular sus procedimientos propios para aprender. 


\section{Conclusiones}

La investigación tuvo lugar en la institución educativa 1094 del distrito de Magdalena del Mar, Trujillo, Perú. Su objetivo fue conocer el estado del uso del número en el proceso de enseñanza - aprendizaje con menores de cinco años. Se emplearon métodos como la entrevista, el diario y la observación y técnicas como el análisis documental y la triangulación; todos desde un enfoque cualitativo y permitieron llegar a las siguientes conclusiones:

En el análisis diagnóstico se evidenciaron diversas formas de actuar en el personal docente participante en la investigación, una parte con un actuar comprometido, y otros con un actuar rutinario, el primero deja notar el pensamiento crítico y reflexivo del profesorado en su práctica educativa, además del testimonio de actividades innovadoras, y alegría al ver los logros de sus estudiantes en el aula; el segundo se evidenció en el trabajo del aula en donde pierde relevancia el análisis al juego y los comportamientos variados, así como el rescate de comunicaciones orales relevantes al uso del número, lo que dificulta retroalimentar y mejorar estrategias y, como con secuencia, mejorar la práctica educativa.

El personal docente con un actuar rutinario utiliza estrategias no funcionales, trabaja en su mayoría de manera ajena al entorno cultural en donde el alumnado convive. Contradictoriamente, los grupos de infantes se muestran entusiastas y asombrados de ver tantas cosas para explorar, para jugar, para manipular; dos posiciones, que conviven en una misma escuela cuya demanda es la búsqueda de una educación de calidad.

En el transcurso de esta investigación se constató la opinión de los padres y madres, en donde reflejaron contradicciones e información limitada sobre el número, pero se notaba la expectativa por el avance de los niños y niñas en el área matemática. Fue notoria las experiencias que cuentan con respecto al manejo del conteo, la comparación de cantidades, la lectura de carteles con información numérica, en el apoyo para la preparación al primer grado, donde brindan a los niños y niñas materiales para la escritura de números con la ayuda de libros de textos sobre ejercicios con números, sumas y usando cuadernos. Las familias reclaman orientación para acompañar el aprendizaje de sus niños y niñas desde el hogar. 
El uso del número, en estas aulas de educación inicial, de manera transversal y lúdica, tiene una connotación limitada; sin embargo, es una potencialidad necesaria que debe ser explotada a través de estrategias pertinentes.

\section{Referencias}

Alsina, A. (2012). Hacia un enfoque globalizado de la educación matemática. Números, 80(2), 24-30.

Castro, E., Cañadas, M., y Castro, E. (2013). Pensamiento numérico en edades tempranas. Artículo. Recuperado de https://dialnet.unirioja.es/descarga/articulo/4836753.pdf

De Castro, C. (2007). La evaluación de métodos para la enseñanza y el aprendizaje de las matemáticas en la educación infantil. Artículo. Recuperado de www.fisem.org/www/union/revistas/2007/11/ Union_011_008.pdf

Bisquerra, R. (1996). Métodos de investigación educativa. Guía práctica (Edición revisada). Barcelona: CEAC.

Carraher, T., y Carraher, D. (2004). En la vida diez, en la escuela cero ( $9^{\text {na }}$ ed.). Sao Paulo, Brazil: Siglo XXI.

Rencoret, M. (1995). Iniciación matemática. Un modelo de jerarquía. Mexico: Andrés Bello.

Chamorro, M. D., Ruiz Higueras, M. L., y Vecino, F. (2005). Didáctica de las matemáticas para educación inicial. Madrid, España: Pearson.

Chamorro, M. C. (2011). La mejora del aprendizaje del área lógico-matemática desde el análisis del currículum de educación infantil. Educación Siglo XXI, 29(2), 23-40.

D'Ambrosio, U. (2014). Las bases conceptuales del Programa Etnomatemática. Revista Latinoamericana de Etnomatemática, 7(2), 100-107.

De la Rosa, V. (2012). Proyectos vivenciales en un aula multigrado. Una propuesta integral de aprendizaje hacia la gestión de aprendizaje en escuelas de $1^{\text {er }}$ grado. En Memorias del proyecto "Intercambio y Socialización de Experiencias Exitosas en el Contexto Escolar" del Instituto Estatal de Evaluación e Innovación Educativa. México. Recuperado de http://ineval.chiapas.gob.mx/ pdf/calidad/exper/mem5/memoria5.pdf. 
Farci, G. (2007). Guía ilustrada como recurso didáctico para determinación "in situ" de algunas macro algas de Playuelita Parque Nacional Morrocoy. Revista Laurus, 13, 86-94.

Figueroa, R. (2012). Competencias matemáticas según género en una institución educativa del Callao (Tesis de maestría). Universidad San Ignacio de Loyola, Perú.

Gonzalez, A., y Weintein, E. (2013). Enseñanza de la matemática. Lima, Perú: Centauro.

Hernandez, J. (2013). El juego como estrategias para adquirir las nociones de conteo y favorecer la noción del número. INVEDU, 7(19), 50-51. Recuperado de http://universidadlasallebenavente. edu.mx/investigacion/revistas/ULSAB_RevInvEdu_19.pdf

Hilber, B., y Higuita , C. (2014). Una mirada a la etnomatemática y la educación matemática en Colombia: Caminos recorridos. Revista Latinoamericana Etnomatemática, 7(2), 245-269.

Knijink, G. (1 de abril de 2014). Etnomatemática en movimiento, perspectivas etnomatemáticas sus formaciones teóricas y ejemplificaciones. Revista Latinoamericana Etnomátematica, 7(2), 222-244.

Ministerio de Educación. MINEDU. (2009). Diseño curricular nacional. Lima: Minedu.

Ministerio de Educación. MINEDU. (2015). Aprendizaje del primero al sexto. Un estudio longitudinal en instituciones educativas de Lima Metropolitana (Serie Investigaciones). Lima, San Borja, Perú: Técnica.

Ministerio de Educación. MINEDU. (2015). Rutas del aprendizaje. Lima: Minedu.

Nuñez. (2010). Inicio de una investigación de diseño sobre el desarrollo de competencias numéricas con niños de 4 años. Madrid, España: Universidad Complutense de Madrid.

Ortiz, P., y Pastor, C. (2014). Desafios de la educación prescolar, básica y media en América Latina (K. Adenauer, Ed.). Santiago de Chile, Chile: Sopla.

Piaget, J. (1991). Seis estudios de sicología (V. Jordi (Ed.) y M. Jordi (Trad.). Barcelona, España: Labor.

Pinto, N., Giron, G., y Villegas, W. M. (2014). Los valores de la familia en la educación inicial. Universidad Pedagógica Experimental Libertador (IPMAR) Maracay - Venezuela. 
Puig, L. (1995). Semiótica y matemáticas (Vol. 51). Valencia, Valencia, España: LLICSO.

Salas, A. (2012). Programa Jugando en los sectores para desarrollar capacidades matemáticas (Tesis de maestría). Universidad San Ignacio de Loyola, Perú.

Swick, K., y Willians, R. (5 April 2006). Analysis of Bronfenbrenner's Bio-Ecological Perspective for Early Childhood Educators: Implications for Working with Families Experiencing tress. Early Chilhood Educations Journal, 33(5), 371-380.

Taylor, S. J. y Bogdan, R. (1990). Introducción a los métodos cualitativos de investigación. . La búsqueda de los significados. Buenos Aires: Ediciones Paidós Ibérica.

Ugartetxea. J. (2001). Motivación y metacognición, más que una relación. RELIEVE, 7(2), 51-71. Recuperado de http://www.uv.es/ RELIEVE/v7n2/RELIEVEv7n2_1.htm

Woods, P. (1993). La escuela va por dentro. La etnografia en la investigación cualitativa ( $3^{\mathrm{a}}$ ed.). Madrid: Ediciones Paidós Ibérica. 